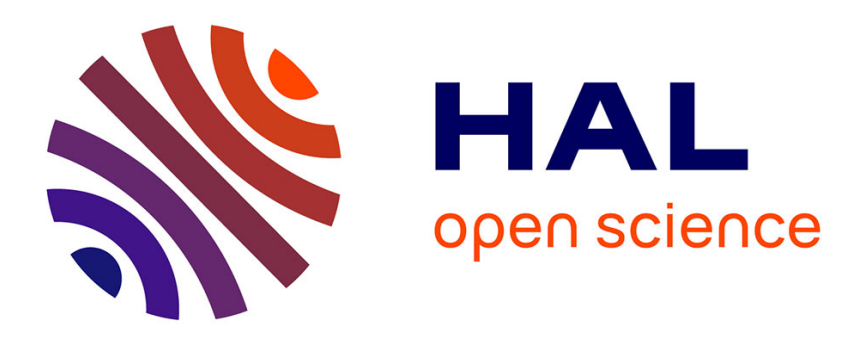

\title{
Trapping microfluidic drops in wells of surface energy
}

Rémi Dangla, Sunyon Lee, Charles N. Baroud

\section{To cite this version:}

Rémi Dangla, Sunyon Lee, Charles N. Baroud. Trapping microfluidic drops in wells of surface energy. Physical Review Letters, 2011, 107 (12), pp.124501. 10.1103/physrevlett.107.124501 . hal-00998003

\section{HAL Id: hal-00998003 \\ https://hal-polytechnique.archives-ouvertes.fr/hal-00998003}

Submitted on 8 Jul 2014

HAL is a multi-disciplinary open access archive for the deposit and dissemination of scientific research documents, whether they are published or not. The documents may come from teaching and research institutions in France or abroad, or from public or private research centers.
L'archive ouverte pluridisciplinaire HAL, est destinée au dépôt et à la diffusion de documents scientifiques de niveau recherche, publiés ou non, émanant des établissements d'enseignement et de recherche français ou étrangers, des laboratoires publics ou privés. 


\title{
Trapping Microfluidic Drops in Wells of Surface Energy
}

\begin{abstract}
Rémi Dangla, Sungyon Lee, and Charles N. Baroud*
LadHyX and department of Mechanics, Ecole Polytechnique, CNRS, 91128 Palaiseau Cedex, France (Received 15 March 2011; revised manuscript received 1 August 2011; published 15 September 2011)

A small hole etched in the top of a wide microchannel creates a well of surface energy for a confined drop. This produces an attractive force $F_{\gamma}$ equal to the energy gradient, which is estimated from geometric arguments. We use the $\operatorname{drag} F_{d}$ from an outer flow to probe the trapping mechanism. When $F_{d}<F_{\gamma}$, the drop deforms but remains anchored to the hole. Its shape provides information about the pressure field. At higher flow velocities, the drop detaches, defining a critical capillary number for which $F_{d}=F_{\gamma}$. The measured anchoring force agrees with the geometric model.
\end{abstract}

DOI: 10.1103/PhysRevLett.107.124501

PACS numbers: 47.55.D-, 47.15.gp, 47.61.-k

When a spherical drop enters a channel whose height is smaller than the sphere diameter, the drop must squeeze and depart from its relaxed shape. The resulting deformation leads to an increase in its interfacial area $A$ and a corresponding increase in free energy $E_{\gamma}=\gamma A$, with $\gamma$ the interfacial tension. By this mechanism, the drop can store and transport this extra energy as it travels down the channel. Given the chance, it will tend to decrease its surface area in order to reduce its free energy. Variations in the level of confinement can therefore be used to apply a force on the drop by inducing gradients of the surface energy. The migration of droplets in such gradients has been known since Hauksbee [1] and exploited in several geometries [2-5], both for wetting and nonwetting drops.

A novel use of surface energy gradients was recently demonstrated in the context of microfluidics [6]. It consists of creating localized areas of reduced confinement by etching grooves into the top surface of a microchannel, in order to attract drops to particular locations. It was shown that the induced energy gradients are able to guide or anchor drops against a mean flow if the outer flow velocity is below a critical value, much as oranges are trapped at the bottom of a bowl by the gradient of gravitational potential energy.

We now turn to the physics behind such anchoring, namely, the balance between the force due to surface energy gradient $\left(\vec{F}_{\gamma}\right)$ and the hydrodynamic drag force $\left(\vec{F}_{d}\right)$ due to the outer flow of oil. First, we estimate $F_{\gamma}$ from the geometric study of a static drop. Then, we consider the conditions for which a drop remains anchored to the hole, namely $F_{d}<F_{\gamma}^{*}$ with $F_{\gamma}^{*}$ the maximum force due to surface energy gradient. We analyze the shape of the elongated drops and extract a scaling law for the $\operatorname{drag} F_{d}$. Last, we measure the threshold velocity above which the drop cannot be anchored, for which $F_{d}=F_{\gamma}^{*}$. This yields a direct measure of the maximum strength of the anchoring mechanism.

The experiments were conducted in polydimethylsiloxane (PDMS) microchannels (Dow Corning Sylgard 184), fabricated using dry film photoresist soft lithography techniques [6,7]. The microchannel consists of a flow-focusing junction to generate droplets, connected to a test section (width $=3 \mathrm{~mm}$ ) containing a single anchor of diameter between $d=50$ and $82 \mu \mathrm{m}$ and of depth between $e=28$ and $45 \mu \mathrm{m}$, as sketched in Fig. 1(a). The channel height is constant everywhere else and ranges from $h=28$ to $60 \mu \mathrm{m}$.

The oil is injected with two syringe pumps, at flow rates $Q_{f}$ in the flow-focusing junction and $Q_{o}$ through the entrainment channel [see Fig. 1(a)]. By injecting water at a flow rate $Q_{w}$, water droplets are formed and transported into the test section. Once a drop is trapped by the single anchor, the water flow is set to zero and $Q_{o}$ is gradually increased until the droplet detaches at a critical flow rate $Q^{*}$. The experiment is repeated for different drop and channel parameters.
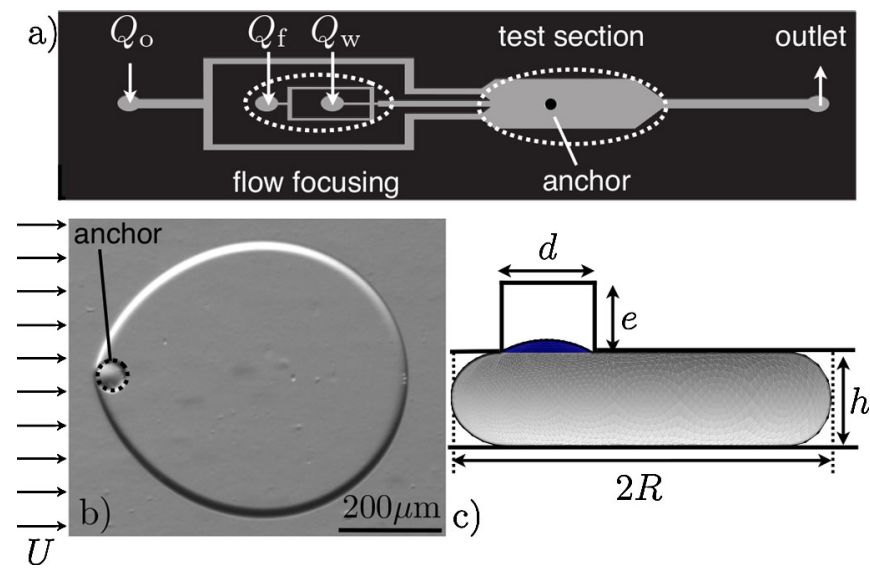

FIG. 1 (color online). (a) Schematic of a microchannel that consists of a flow focuser to generate water drops in oil and of a test section with a single anchor. (b) A top-down image of a pancake droplet held in place by an anchor against a mean external flow $U$. (c) Surface Evolver rendering of an anchored droplet of outer radius $R$ inside the microchannel of height $h$ over an anchor of diameter $d$ and of depth $e$. 
The continuous phase consists of a fluorinated oil (3M Fluorinert FC-40 or FC-70 of viscosities $\mu \approx 4.1 \mathrm{cP}$ and $\mu \approx 24 \mathrm{cP}$ at $25^{\circ} \mathrm{C}$, respectively). Contrary to alkanes or silicone oils, these oils do not swell the PDMS and the surfaces of the channel remain parallel even for large aspect ratios [8]. One of two different fluorosurfactants, based on the molecules described in Ref. [9], is added to the oil phase at a concentration of $2 \%$ : the first is a PFPEPEG molecule while the second has a simpler ammonium salt head group [9]. The water-oil interfacial tension $\gamma_{e}$ is measured via the pendant drop method. Independently of the oil type, the equilibrium value is found to be $\gamma_{e}=20 \mathrm{mN} / \mathrm{m}$ and $\gamma_{e}=10 \mathrm{mN} / \mathrm{m}$ for the PFPE-PEG and ammonium salt surfactants, respectively. The time to reach these equilibrium values ranges from 30 minutes for the first surfactant, up to several hours for the second.

As illustrated in Fig. 1(b), even small anchors are able to hold relatively large droplets. This implies that a droplet reduces its overall surface area, despite locally creating excess surface as it partially enters into the anchor. This can be verified by comparing the surface area of a circular pancake drop, in the absence of an anchor, to the modified area when the drop is above the anchor. The area of the unperturbed drop is estimated by modeling it as a circular cylinder of radius $R_{i}$, surrounded by the outer half of a torus of small radius $h / 2$. This is strictly valid in the limit of $\varepsilon \equiv h / 2 R_{i} \ll 1$ and yields the area of the drop: $A_{i}=2 \pi R_{i}^{2}[1+\pi \varepsilon]$.

When a static droplet feels the presence of an anchor, it penetrates into it and forms a spherical cap whose local curvature equilibrates with the interface curvature far away. The parameter $b \equiv d / h$ determines the behavior of the drop: If $b>2$, curvature equilibrium is reached only when the drop enters fully into the hole and the spherical cap flattens against the top, rendering the hole depth $e$ an important parameter. Here, we will limit ourselves to the case of $b \leq 2$, in which the drop only partially enters into the hole. This situation is depicted in Fig. 1(c), which features a Surface Evolver simulation of the drop shape in the absence of flow [10]. By matching the curvature of the spherical cap in the hole with the curvature of the interface far away $\mathcal{C} \approx 2 / h+\pi /(4 R)$ [11] and imposing the volume conservation of the droplet, a new value of $R$ can be obtained. This yields a new value of the surface area of the drop $A$ in the presence of the anchor. The change in surface area $\Delta A=A-A_{i}$ then corresponds to

$$
\Delta A=-\left[\frac{\pi}{2} b S(b)+O(\varepsilon)\right] h^{2},
$$

where

$$
S(b) \equiv \frac{b}{2}-\frac{4}{3 b}\left[1-\left(1-\frac{b^{2}}{4}\right)^{3 / 2}\right] .
$$

The dimensionless function $S(b)$ is positive for $0<b \leq 2$, resulting in a negative $\Delta A$ and a decrease in
$E_{\gamma}$. Therefore, even an asymptotically small anchor reduces the droplet surface energy, yielding $\Delta E_{\gamma}=\gamma \Delta A<0$. For $b>2$, the energy change remains negative but its value will depend on the hole depth; $S(b)$ does not fully capture the energy change in this case.

The magnitude of the force due to this change in surface area is equal to the gradient of surface energy. Estimating it therefore requires an estimate of the distance over which the energy changes. Figure 1(b) shows that even a weak external flow aligns the edge of the drop with the anchor. This indicates that the gradients of surface energy appear only as the edge of the drop passes over the hole. Therefore, the characteristic length over which the energy changes is given by the hole diameter $d$ and the force can be estimated as

$$
F_{\gamma}^{*} \propto \frac{\left|\Delta E_{\gamma}\right|}{d} \approx \gamma \frac{\pi}{2} h S(b) .
$$

Having determined the anchoring force based on the static droplet geometry, we now consider the drag force $\vec{F}_{d}$, exerted by the outer flow, which tends to push the drop out of the anchor. Given the scale separation between the vertical $(z)$ and in-plane $(x, y)$ dimensions, the test section of the channel is modeled as a Hele-Shaw cell [12]. Hence, the height averaged velocity field $\vec{U}$ is potential and verifies Darcy's law

$$
\vec{\nabla} p=-\frac{12 \mu}{h^{2}} \vec{U},
$$

where the pressure $p$ is invariant in the vertical direction.

The drag force $\vec{F}_{d}$ acting on the droplet has two components: the pressure drag $\vec{F}_{p}=-\int p_{o} \vec{d} s$, defined as the outer pressure $p_{o}$ at the interface applied on the surface elements $\vec{d} s$, and the viscous $\operatorname{drag} \vec{F}_{\mu}=\int \mu \vec{\nabla} \vec{U} \cdot \vec{d} s$. Darcy's law (4) provides an estimate for the pressure as $p \propto \mu U R / h^{2}$, while viscous shear stresses $\mu \vec{\nabla} \vec{U}$ scale as $\mu U / h$ to the leading order. Since both the pressure and shear stresses act on the lateral drop surface $(\propto h R)$, the pressure drag $F_{p} \propto \mu U R^{2} / h$ is greater than the viscous $\operatorname{drag} F_{\mu} \propto \mu U R$ by an order $R / h \gg 1$. One may therefore ignore the viscous drag and focus on the pressure drag.

This scaling argument has been validated by asymptotic analyses of the Hele-Shaw flow around a stationary inviscid bubble [13] and around a solid cylinder [14]. Those studies confirm the dominant role of pressure drag and provide expressions for $p_{o}$ and $\vec{F}_{d}$ as

$$
\begin{aligned}
& p_{o}=-24 \frac{\mu U}{h^{2}} x+\mathrm{Cst}, \\
& \vec{F}_{d}=24 \pi \frac{\mu U R^{2}}{h} .
\end{aligned}
$$

In order to verify this expression for $p_{o}$, we analyze the shape of anchored droplets. Indeed, the Laplace equation gives a local relationship between the mean curvature of 
the drop $\mathcal{C}$ and the pressure jump, $p_{i}-p_{o}$, across the interface. This provides an optical readout of the pressure variations through the drop shape. By defining $\delta r(\theta)$ as the local deviation from the radius $R$ in the absence of flow (see Fig. 2), the Laplace equation reduces to

$$
\gamma\left\{\frac{2}{h}+\frac{\pi}{4} \frac{1}{R}\left[1-\frac{1}{R}\left(\delta r(\theta)+\frac{d^{2} \delta r}{d \theta^{2}}\right)\right]\right\}=p_{i}-p_{o},
$$

in the limit of $\delta r / R \ll 1$ [11].

Although Eq. (6) involves the pressure field within the droplet $p_{i}$, this field does not affect the deformations. In fact, the internal flow must satisfy potential flow theory to leading order and, given the static drop interface as the boundary, the only possible velocity field is the trivial $\vec{U}=\overrightarrow{0}$ with a constant $p_{i}$. Consequently, the shape deformations $\delta r(\theta)$ are mainly determined by the outer pressure $p_{o}$. Then, inserting the expression for $p_{o}$ from Eq. (5a) in Eq. (6) yields

$$
\frac{\delta r(\theta)}{R}=15.3 \mathrm{Ca} \frac{R^{2}}{h^{2}}(1-\theta \sin \theta) .
$$

Equation (7) provides a scaling law for the drop elongation $L \equiv \delta r(0) / R \propto \mathrm{Ca} R^{2} / h^{2}$. This scaling is verified in Fig. 2 where $L h^{2} / R^{2}$ is plotted vs Ca for various channel geometries, liquid viscosities, surfactant types, and oil flow

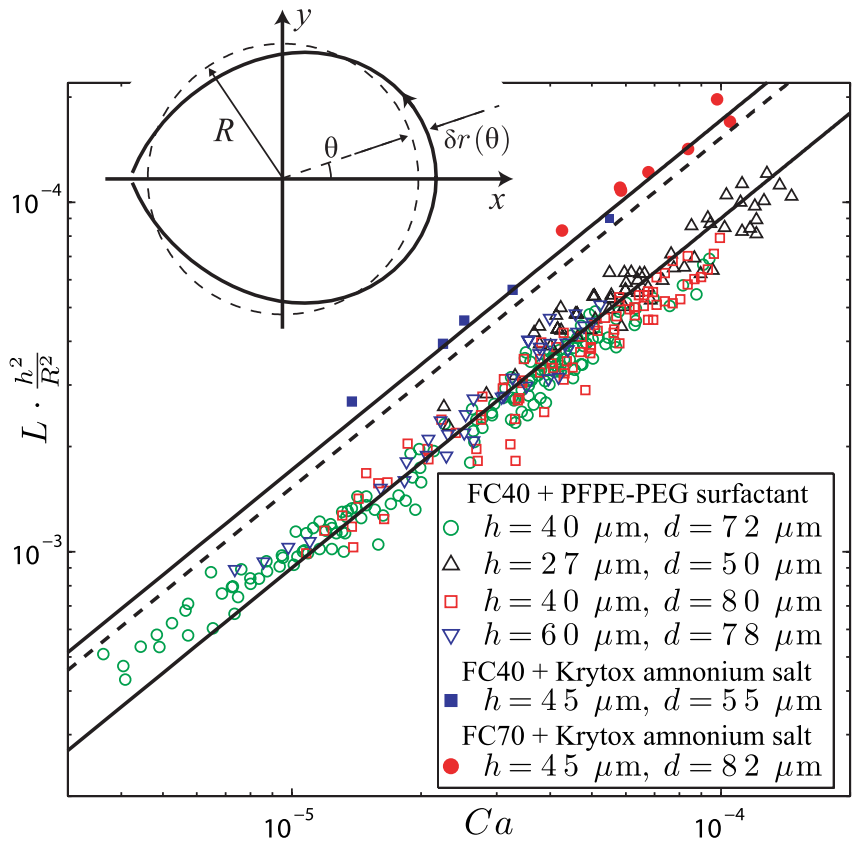

FIG. 2 (color online). Inset: Sketch of the polar coordinates for the deformations $\delta r(\theta)$ from the static radius $R$. Main panel: Elongation $L=\delta r(0) / R$ of anchored droplets, rescaled by $h^{2} / R^{2}$, as a function of the oil capillary number $\mathrm{Ca}$ for various channels, liquids, and surfactants. The solid lines are a linear fits of the two data groups, with slopes $9 \pm 2$ (PFPE-PEG surfactant) and $17 \pm 1$ (ammonium head group). The dashed line is the theoretical prediction of Eq. (7), with slope 15.3. rates $Q_{o}$ [or equivalently flow velocities $U_{o}=Q_{o} /(W h)$ ]. The data collapse onto two distinct lines which both verify the linear scaling, although with different prefactors. The value of the prefactor is only a function of the surfactant type and is independent of the other parameters.

Since the two surfactants exhibit different adsorption kinetics in a pendant drop experiment, they may also reach different spatial distributions on the drop interface in the presence of an external flow [15]. Indeed, complex recirculation patterns are observed on the surface of our droplets, indicating that Marangoni stresses are present on the interfaces. The dependence of these stresses on the surfactant kinetics could explain the different prefactors that are observed in the elongation experiments.

Nevertheless, the drop shape is well described by Eq. (7), once the prefactor 15.3 is replaced by the slope of the linear fit from Fig. 2. The predicted deformations display a good agreement with the drop shape at different flow rates, as illustrated in Fig. 3. Given that the pressure deforming the drop is linear in $x$, the drop shape obtained in this flow is akin to a $2 \mathrm{D}$ pendant drop, where the hydrostatic pressure also decreases linearly with height.

The elongation and shape measurements of Figs. 2 and 3 verify the amplitude of the pressure variations along the drop and, thereby, the scaling for the pressure drag in Eq. (5b): $F_{d} \propto \mu U R^{2} / h$. We can now obtain the anchor strength by finding the maximum oil velocity $U^{*}$ that the anchored droplet can resist and by using the force
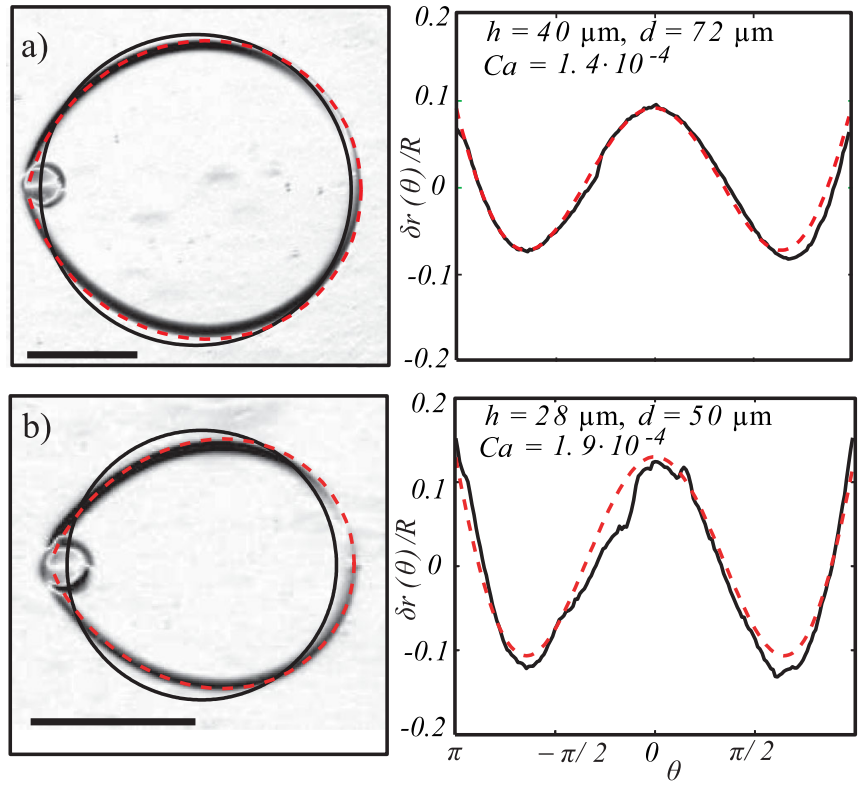

FIG. 3 (color online). Left: Images of anchored droplets deformed by the flow of oil. The dashed lines show the prediction from Eq. (7), while the solid line shows the equivalent circle of radius $R$. Scale bars represent $200 \mu \mathrm{m}$. Right: Comparison between the deformations $\delta r(\theta) / R$ extracted from the microscope images on the left (solid line) and the theoretical curve (dashed line) obtained from Eq. (7), using 9 as a prefactor. 


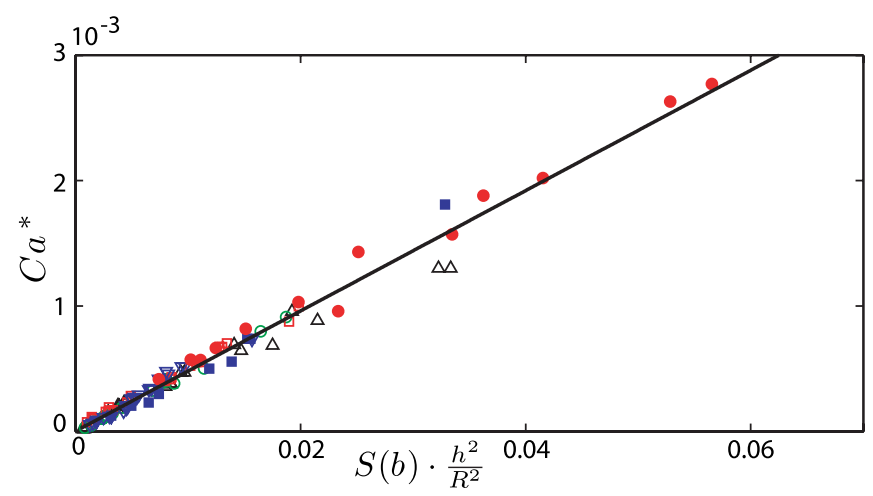

FIG. 4 (color online). Critical capillary number $\mathrm{Ca}^{*}$ plotted against $S(b) h^{2} / R^{2}$ for the channel geometries, liquids, and surfactants as listed in the legend of Fig. 2. The solid line is a linear fit of the data.

equilibrium $F_{\gamma}^{*}=F_{d}\left(U^{*}\right)$. This yields the scaling

$$
\mathrm{Ca} * \propto S(b) \frac{h^{2}}{R^{2}},
$$

where $\mathrm{Ca}^{*} \equiv \mu U^{*} / \gamma$.

The experimental results agree with this theoretical prediction, as seen in Fig. 4 which displays the value of $\mathrm{Ca}^{*}$ for all channels, liquids, and surfactants. The data all collapse on a single linear law, thus confirming the scaling law (3) for the anchoring force $F_{\gamma}^{*}$, which is derived from purely geometric arguments. In particular, it supports the use of $d$ as the characteristic length scale in $F_{\gamma}^{*}$. The actual value of the anchoring force can be estimated by reintroducing the numerical prefactor of Eq. (5b). It yields values of $F_{\gamma}^{*}$ in the range of $100-1000 \mathrm{nN}$, depending on the geometric parameters $h$ and $d$.

These results verify that the gradient of surface energy and the hydrodynamic drag due to the external fluid are indeed the dominant competing physical mechanisms that determine the ability to anchor a droplet. In this respect, anchoring droplets in wells of surface energy resembles other methods of trapping in energy wells, such as electrostatic cups [16] or optical tweezers [17], where the maximum restoring force is given by the maximum energy gradient. Below this limit, deviations from the equilibrium shape or position provide information about the magnitude of the opposing forces at play in all three examples. In the present case, the drop shape depends on the surfactant type, in addition to the global force balance, which may yield information about the physical chemistry at the free interface.

An important distinction between the anchors and other trapping mechanisms resides in the source of energy: while most systems rely on an external field, the energy here is contained in the droplet itself. The typical values of surface energy therefore increase the range of attainable forces up to several hundred $\mathrm{nN}$. This value can be further increased by etching larger and deeper holes, thus exploring the regime $b>2$. In this case, the drop extends fully into the hole into a spherical or cigar shape and the gain in surface energy is maximized. Although this limit is not studied here, such devices would exert very large anchoring forces.

In terms of applications, anchors are simple and efficient passive devices to hold drops stationary, even in the presence of an outer flow. Parallelization is straightforward and enables easy production of droplet arrays for chemical or biomedical studies. The array format has gained in popularity recently since it provides a direct microfluidic equivalent to classic multiwell plates which are ubiquitous in biological applications. A key benefit over existing methods is the continuous flow of outer liquid, which can be used to control the chemical environment of the trapped droplets and their content, both in space and time. A wide range of chemical and biomedical applications follows, from protein crystallization to the study of sickle cell anemia [6]. Finally, more controlled droplet placing can be achieved by combining the passive "rails and anchors" approach with active forcing of individual droplets, for example, using a focused laser [18].

We thank François Gallaire and Mathias Nagel for fruitful discussions, and Caroline Frot for the microfabrication. Estelle Mayot provided invaluable surfactant help and Wiebke Drenckhan and Anniina Salonen helped with the surface tension measurements. S. L. was funded by a Chateaubriand fellowship and the Direction des Relations Extérieures of Ecole Polytechnique.

*baroud@ladhyx.polytechnique.fr

[1] F. Hauksbee, Phil. Trans. R. Soc. London 27, 395 (1710).

[2] É. Lorenceau and D. Quéré, J. Fluid Mech. 510, 29 (2004).

[3] T. Metz, N. Paust, C. Muller, R. Zengerle, and P. Koltay, Sens. Actuators A, Phys. 143, 49 (2008).

[4] P. Renvoisé, J. Bush, M. Prakash, and D. Quéré, Europhys. Lett. 86, 64003 (2009).

[5] T. Metz, N. Paust, R. Zengerle, and P. Koltay, Microfluid. Nanofluid. 9, 341 (2009).

[6] P. Abbyad, R. Dangla, A. Alexandrou, and C. N. Baroud, Lab Chip 11, 813 (2011).

[7] K. Stephan, P. Pittet, L. Renaud, P. Kleimann, P. Morin, N. Ouaini, and R. Ferrigno, J. Micromech. Microeng. 17, N69 (2007).

[8] R. Dangla, F. Gallaire, and C. N. Baroud, Lab Chip 10, 2972 (2010).

[9] J. Clausell-Tormos et al., Chem. Biol. 15, 427 (2008).

[10] K. Brakke, Surface Evolver manual, http://www .susqu.edu/facstaff/b/brakke/evolver/evolver.html (2008).

[11] C. Park and G. Homsy, J. Fluid Mech. 139, 291 (1984).

[12] G. I. Taylor and T. G. Saffman, J. Appl. Math. Mech. 12, 265 (1959).

[13] S. Maruvada and C. Park, Phys. Fluids 8, 3229 (1996).

[14] J. Lee and Y. Fung, J. Fluid Mech. 37, 657 (1969).

[15] V. Levich, Physicochemical Hydrodynamics (Prentice Hall, Englewood Clifs, NJ, 1962).

[16] M. Krishnan, N. Mojarad, P. Kukura, and V. Sandoghdar, Nature (London) 467, 692 (2010).

[17] A. Ashkin, Proc. Natl. Acad. Sci. U.S.A. 94, 4853 (1997).

[18] E. Fradet et al., Lab Chip (to be published). 